\title{
FORMAÇÃO DE ESTRATÉGIA NAS MICRO E PEQUENAS EMPRESAS: UM ESTUDO NO CENTRO-OESTE MINEIRO
}

\section{RESUMO}

Muito se tem dito e escrito sobre as peculiaridades da administração estratégica nas micro e pequenas empresas (MPE). Pouco, entretanto, tem sido feito em termos de trabalho empírico, de cunho quantitativo, quando comparado com o volume de pesquisa que trata de grandes empresas. N esse sentido, 0 presente artigo buscou explorar o processo de formação de estratégias nessas empresas, descrevendo seus principais aspectos e, principalmente, verificando se há características desse processo que podem ser associadas a níveis de desempenho acima (e/ou abaixo) da média. Para tanto, utilizou-se a análise de regressão linear múltipla para entender as relações existentes entre as variáveis do processo de formação da estratégia consideradas no estudo e o desempenho; e, ainda, a análise discriminante para determinar os indicadores que diferenciam os grupos de empresas com desempenho acima e abaixo da média. Os resultados apontaram uma tendência à utilização de processos de formação de estratégia deliberados e formais. Por outro lado, reconhecemos também, uma tendência de inovação e mudança. 0 desempenho acima da média das micro e pequenas empresas, ao que parece, é resultado de uma receita balanceada desses dois ingredientes.

\section{Leonardo Lemos da Silveira Santos}

Universidade Federal de Lavras e PUC Minas

\section{Ricardo César Alves}

PUC Minas

\section{Kenneth Nunes Tavares de Almeida}

Universidade Federal de Lavras e Faculdades Jorge Amado

\begin{abstract}
M uch has been said on the peculiarities of strategic management in micro and small companies, however, little has been done regarding empirical, quantitative work, when compared with the amount of research that addresses large companies. The present work explores the process of strategy formation in these companies and describes their main aspects. A verification is carried out to ascertain if there are characteristics of this process that can be associated to performance levels. M ultiple linear regression analysis was used to better understand the existing relationships between variables of the same process of strategy formation considered in the study and the performance; the discriminating analysis was used to determine the indicators that distinguish the groups of companies with performance above and bel ow the average. The results pointed a trend to the utilization of deliberate and formal processes of strategy formation. On the other hand, we also recognize a tendency of innovation and change. The performance above average of the micro and small companies, what it seems, is resulted of a balanced prescription of these two ingredients.
\end{abstract}

PALAVRAS-CHAVE Formação de estratégias, desempenho, micro e pequenas empresas, processos, inovação.

KEMORDS Strategy formation, performance, micro and small companies, process, innovation. 


\section{INTRODUÇÃO}

A proposta deste trabalho é identificar e analisar o processo de formação de estratégia nas micro e pequenas empresas, não apenas para descrever o processo, mas também para analisar as relações existentes entre o processo e o desempenho dessas empresas - acima ou abaixo da média de desempenho. As micro e pequenas empresas possuem algumas características que Ihes são próprias e que as tornam essenciais ao funcionamento tanto das economias desenvolvidas quanto daquelas em processo de desenvolvimento.

De maneira geral, as pequenas empresas oferecem significativa contribuição na geração global do produto nacional; e têm alta absorção de mão-de-obra a baixo custo; atuação complementar aos empreendimentos de grande porte; atuação estratégica no comércio exterior, possibilitan do a diversificação na pauta de exportações e tornando a economia menos suscetível às variações que ocorrem na conjuntura comercial mundial; e capacidade de gerar uma classe empresarial genuinamente nacional, aumentando a participação da economia privada na economia do país (Lakatos, 1997).

Contudo, apesar da grande importância que as micro e pequenas empresas têm, nota-se um interesse ainda pequeno no sentido de conhecer, analisar e propor alternativas para esse segmento empresarial. Supostamente, duas das razões para que esse esforço se intensifique são a importância econômico-social da pequena empresa e a crescente evidência dos problemas e dificuldades comuns a essas empresas, os quais são responsáveis pel o seu elevado índice de mortalidade (Figueiredo e M artinelli, 2002, Silva e Pereira, 2004).

Cher (1990) observa que, a despeito do grau de industrial ização ou de desenvolvimento, as pequenas empresas têm papel fundamental na evolução da sociedade, contribuindo tanto do ponto de vista econômico quanto do social e até político. Ao discorrer sobre as características e o rel evante papel que representam para a economia, 0 autor aponta que a pequena empresa apresenta melhor desempenho em atividades que demandam habilidades ou serviços especializados, como desenvolver trabalhos mais personalizados e específicos. Isso Ihes permite obter vantagens sobre as empresas de grande porte. Outro fator que confere uma singularidade às pequenas empresas é 0 fato de conseguirem reagir rapidamente de acordo com a evolução das condições do ambiente, pelo fato de estarem concentradas próximas aos seus mercados.

Entretanto, para Lakatos (1997), o maior problema que aflige as pequenas empresas é sua estrutura demasia- damente centralizada na pessoa do dono ou proprietário, que, geralmente, possui uma baixa qualificação. Assim, nessas empresas inexiste a figura do executivo responsável pelo gerenciamento do negócio. Não menos importante, Lakatos cita também que a divisão de tarefas não se apresenta nítida, havendo acú mulo de funções devido à distribuição deficiente das atribuições. Adicionalmente, outros fatores devem ser considerados, quando se trata das especificidades apresentadas às pequenas empresas. Pinheiro (1996) aponta os desafios operacionais, como a pouca capacidade financeira, o que inviabiliza condições de autofinanciamento, etambém a atual ização tecnológica que as distanciam das grandes empresas. Q uanto à atuação de seus dirigentes, continua aquele autor, observa-se que a baixa eficácia gerencial decorre da fal ta de uma postura empreendedora, que dêsustentação a uma moderna tecnologia gerencial, permitin do a inovação e, por conseguinte, a valorização dos recursos disponíveis.

Segundo Cher (1990), estudiosos da área estimam que cerca de $80 \%$ das pequenas empresas não completam o primeiro ano de atividade, e $92 \%$ fecham suas portas ao final de cinco anos de existência. As cau sas desse al to índice de fechamento das micro e pequenas empresas estão sempre relacionadas à sua capacidade estratégica de responder às influências ambientais. A questão crucial que se coloca às pequenas empresas é que, devido à sua estrutura, esses problemas revestem-se de maior amplitude, ou seja, o impacto cau sado nessas organizações é mais contundente do que seria em uma grande empresa.

Assim, a gestão das pequenas e médias empresas sofre interferências diversas associadas à sua estrutura, capacidade produtiva e, principalmente, a suas competências básicas. A construção ea implementação de estratégias no contexto das pequenas e médias empresas merecem uma análise mais cuidadosa, sendo, por esse motivo, o ponto central destetrabalho. A seguir, apresentamos o referencial teórico de estratégias organizacionais. Posteriormente passamos à discussão da estratégia no âmbito das pequenas e médias empresas, partindo a seguir para a construção e formação da estratégia em micro e pequenas empresas selecionadas para compor a amostra deste estudo. Por fim, 0 artigo é final izado com uma seção de resultados e a apresentação de al gumas conclusões.

\section{FUNDAMENTAÇÃO TEÓRICA}

\section{Estratégias organizacionais}

As organizações passam por um período de adaptação às novas regras competitivas do mercado. $\mathrm{Na}$ verdade, $\mathrm{O}$ 
grande desafio está na condução eficaz das organizações num mercado de extrema competitividade. Com isso, o termo estratégia tornou-se um ponto de destaque de todas as empresas, independentemente de seu porte, segmento ou forma de gestão. Henderson (1998) afirma que, quanto mais rico o ambiente, maior o número de competidores e mais acirrada será a competição. Ele afirma ainda que estratégias são revistas para enfrentar mudanças rápidas em relacionamentos competitivos de mercado, ou seja, 0 mercado fica atrativo para muitas empresas que aumentam a competição por meio de diferentes estratégias de atuação. Então, a administração estratégica passa a ser essencial para a continuidade e a longevidade das organizações, como também argumentam Ansoff e McDonnell (1993, p. 15).

E o que realmente significa estratégia? Como as empresas percebem e utilizam estratégias? Whittington (2002) aponta que muitos consultores e teóricos entram em contradição mesmo quanto à definição do que ée do que não é estratégia. M intzberg polemiza ainda mais a discussão: “[...] estratégia, na verdade, é uma dessas palavras que as pessoas definem de um jeito e usam de forma diferente, sem perceber a diferença" (M intzberg, 2001, p. 186). Essa definição de M intzberg aponta para a dificuldade de se compreender formal mente o que é estratégia. A idéia de estratégia fica pulverizada por sua utilização em transações rotineiras das organizações. Embora as transações de mercado sejam relevantes no contexto organizacional, as firmas não podem ser reduzidas a elas, pois, segundo Chandler (1992), é no interior da organização que funciona a "estufa" para o cultivo de competências inovadoras e duradouras.

Buscando uma definição básica de estratégia, pode-se remeter a Henderson (1998), que define a estratégia como uma busca deliberada de um plano de ação para desenvolver eajustar a vantagem competitiva de uma empresa. Essa vantagem de competição deve ser significativa em rel ação às empresas que exploram o mesmo mercado. Ohmae (1998) destaca que a estratégia deve ser utilizada quando se quer oferecer valor para o consumidor. Porter (1999) complementa a idéia dizendo que a base de uma estratégia competitiva é ser diferente, de modo que os consumidores percebam suas ações como al go diferenciado. A estratégia deve, pois, buscar ( por meio de planos de ação) criar vaIores que diferenciem a organização de seus concorrentes, criando, assim, uma vantagem competitiva.

Porter (1999) salienta ainda que qualquer vantagem competitiva é sustentável até que os concorrentes consigam superar as ofertas de uma dada empresa e criar valor superior àqueles desenvolvidos por essa mesma empresa.
Segundo Prahalad (1998), a curto prazo a competitividade de uma empresa se origina de seus atributos de preço/ desempenho em produtos existentes, mas, a longo prazo, a competitividade deriva da capacidade de formar, a custos baixos e tempo mínimo, competências que propiciem produtos inovadores e que não possam ser copiados a curto prazo. Considerando as definições propostas por Ansoff e M cdonell (1993), que conceituam estratégia como um conjunto de padrões de tomada de decisão para orientação do comportamento de uma organização, e as de Porter (1989), que orienta as ações empresariais para a aquisição de al guma vantagem competitiva, obtém-seuma definição satisfatória. D essa forma, a estratégia pode ser definida como um conjunto de padrões e comportamentos de uma organização para alcançar uma vantagem competitiva por meio da diferenciação de produtos e serviços oferecidos ao mercado.

Meirelles (2003) acrescenta que "[...] estratégia é a criação de uma posição competitiva exclusiva e valiosa, envolvendo um conjunto diferente e compatível de atividades; [...] é exercer opções excludentes na competição; é escolher o que fazer, mas fundamental mente o que não fazer" (Meirelles, 2003, p. 40). Para Saraiva (2004), o amplo debate de representantes de linhas aparentemente antagônicas demonstra que a multidimensionalidade da estratégia torna difícil a possibilidade de haver consenso em torno de uma definição conceitual para ela.

Em síntese, estratégia diz respeito a decisões e ações que impactam o conjunto da organização, lidam com a adequação entre esta e o ambiente de atuação, sendo de natureza complexa e envolvendo aspectos de conteúdo e processo (M eirelles, 2003). N esse sentido, fazer estratégia é conhecer onde e quando essa interação ocorre no tempo e no espaço. A estratégia reuniu o que eram as características básicas de outras áreas da administração para formar os administradores estratégicos (Wilson e Jarzabkowski, 2004). É importante destacar que esse debate acerca da estratégia não se limita às grandes corporações, mas se estende a todos os tipos de empresas ou instituições, independentemente do porte, ramo de atividade, natureza de operações. Assim, no próximo capítulo, parte-se para uma discussão relacionando estratégia às empresas de pequeno porte, ou melhor, às micro e pequenas empresas.

\section{Formação de estratégias}

A partir de uma idéia básica do que é estratégia, Whittington, (2003) define o caminho dos estudos em estratégia nas organizações. 0 autor entende que não se deve procurar nas empresas o conceito (teórico) de estra- 
tégia, mas antes o modo como ela é formulada e trabalhada. Deve-se pesquisar onde e como a atividade de criação e administração de estratégia realmente é feita. Quem realiza essa atividade? Quais as competências necessárias para essa atividade e como são adquiridas? Q uais as ferramentas comuns e as técnicas de criação eadministração de estratégias? Como os resul tados são divulgados, utilizados e monitorados? Portanto, compreender a estratégia na organização não significa, necessariamente, conceituar ou criar definições, mas enten der o processo interativo entre pessoas, ambiente, organização e estratégia. A pesquisa deve compreender a estratégia como um amplo campo de atividade social cujas práticas são importantes para a sociedade como um todo (W hittington, 2004).

M intzberg e Waters (1985) afirmam que a formação das estratégias ocorre em duas direções: uma deliberada e outra emergente. A diferença fundamental entre a estratégia deliberada e a emergente é que, enquanto a primeira enfatiza o planejamento, a direção e o controle, fazendo com que se realizem intenções, aúltima permite a noção de "aprendizado estratégico", ou seja, as empresas se desenvolvem à medida que 0 ambiente competitivo gera novos cenários de atuação. U ma estratégia pode emergir como resposta a uma situação em evolução, ou pode ser introduzida deliberadamente, por meio de um processo de formulação seguido de implementação. As estratégias emergentes são ações praticadas na dinâmica das organizações, não sendo objeto de planejamento. Posteriormente, uma vez bem-sucedidas, passam a se integrar ao esforço de planejamento da instituição (Mintzberg, 1998).

Para Saraiva (2004), a estratégia seria originada a partir do contexto, entendendo-se por este último o conjunto de variáveis formado por tempo, cultura organizacional, seu ambiente e o nível de estabilidade deste, atividades, estrutura e aparato tecnológico da organização e, ainda, sua liderança e sistema político interno. M intzberg (1998) defende a criação artesanal da estratégia em contrapartida ao desenvolvimento racional e deliberado de planos e padrões de ações. 0 autor enten de que a imagem de uma criação artesanal da estratégia é a que melhor representa o processo de el aboração de uma estratégia eficaz. As estratégias podem combinar claramente del iberação e controle com flexibilidade e aprendizagem organizacional. Sentimento, comprometimento e experiência são mais importantes do que a razão no processo de formulação da estratégia: "[...] os processos de formulação e implementação transformam-se em um processo contínuo de aprendizagem através do qual surgem estratégias criativas" (Mintzberg, 1998, p. 419).
O planejamento e a racionalização na formulação das estratégias são constantemente atacados por M intzberg e por outros autores de estratégia. Porém, Goold e Campbell (1998) apontam que não existe uma maneira única e meIhor de se formularem estratégias: estas são atitudes que dependerão da natureza dos negócios de atuação, das pessoas envolvidas no processo, da necessidade de ação exigida pelo mercado, bem como dos objetivos e estrutura da organização. M as Wack (1998) acredita ser muito perigoso tentar prever uma situação futura num contexto deinstabilidade. Para ele, nenhuma projeção correta pode ser deduzida a partir do comportamento passado, ea meIhor abordagem é aceitar as incertezas: "[...] a incerteza hojenão é apenas um desvio ocasional temporário de al go razoavel mente previsível; é uma característica básica do ambiente de negócios" (Wack, 1998, p. 363).

Segundo M intzberg, Ahlstrand e Lampel (2000, p. 267), “[...] nenhuma estratégia do mundo real pode ser puramente del iberada ou emergente, uma vez que uma impede 0 aprendizado enquanto a outra impede o controle. Assim, a pergunta passa a ser: que grau de cada uma é adequado, onde e quando?". Dessa manei ra, deve-se buscar compreender a importância da del iberação e da emergência para a construção de uma estratégia, havendo lugar para planejamento, intuição, feeling e vários processos cognitivos. A formulação de estratégias nas organizações dependerá da corrente teórica que mais influencie os gestores, e da natureza, complexidade, competição e outros fatores que moldam o ambiente de negócios. A formulação e implementação de estratégia devem considerar aspectos internos da organização e externos, local izados no ambiente, e recomenda-se compatibilizar esses dois conjuntos de elementos ( Meirelles, 2003, p. 50).

A percepção dos vários fatores internos ou externos que compõem o sistema organizacional pode influenciar a formação da estratégia numa organização. N esse caso, faz-se necessário entender também quem é (são) o(s) responsável (is) pela criação e o desenvolvimento de estratégias para identificar a influência dele(s) no processo como um todo. Mintzberg (2001, p. 232) aponta o executivo principal da empresa como o responsável pela estratégica da organização:

[...] a criação da estratégia, naturalmente, é responsabilidade do executivo principal e o processo tende a ser altamente intuitivo, com freqüência orientado para a procura agressiva de oportunidades. Não é de surpreender, portanto, quea estratégia resultantetenda a refletir a visão implícita de que o executivo principal tem do mundo, muitas vezes uma extrapolação de sua personalidade. 
Em relação aos aspectos externos, o ambiente será sempre o ponto de equilíbrio para a orientação estratégica que a organização irá assumir. A avaliação das condições externas e as ações internas que a organização deve adotar em face da complexidade do contexto, e a análise e crítica sobre a efetiva existência de um macroambiente dinâmico, são fatores primordiais na hierarquia de prioridades que a organização deve executar (Alves, 2006). Toda organização, independentemente de seu porte, suas intenções e objetivos, é continuamente submetida a relações que se delineiam em um contexto com um grande número de atores com interesses diversos, constituindo uma dinâmica de conflitos e consensos que invariavelmente se superpõem. Assim, cabe às organizações estarem atentas para ajustar sua gestão, processo que envolve desde a definição de objetivos até a apreciação dos condicionantes ambientais de mercado. Vejamos, a seguir, 0 caso específico da formulação de estratégias em micro e pequenas empresas.

\section{Estratégia em micro e pequenas empresas}

Para M eirelles et al. (2000), a crença de que apenas "boas idéias" seriam suficientes para assegu rar o sucesso e a sobrevivência de uma organização não vem demonstrando ser verdadeira por alguns motivos. Primeiro, devido às falhas nas previsões em que se apóiam os executivos e nas quais suas idéias se baseiam, e segundo, pelo fato de as mudanças ambientais serem tantas que os procedimentos estruturados se revelam insuficientes para adequar a organização à nova realidade. Assim, uma organização, em função de seus valores, sua cultura, sua capacidade financeira e seus objetivos, deve encontrar o mel hor modelo de formulação e implementação das estratégias que fornecerão a base para seu sucesso ou insucesso.

$\mathrm{N}$ a maioria das vezes, as pequenas e médias empresas têm apresentado certas dificuldades que, de acordo com Bal estrin e Vargas (2003), envolvem obstáculos na obtenção de melhores preços e vantagens. Essas dificuldades são encontradas na compra de matérias-primas e componentes, nos custos de participação em feiras, campanhas publicitárias, reciclagem e treinamento da mão-de-obra, atual ização tecnológica, acesso a linhas de crédito e financiamento, aluguéis e final mente os custos envolvidos na exportação de produtos.

Para Cassaroto Filho ePires (1998), o grande problema da pequena empresa é a falta de competência para dominar todas as etapas da cadeia de valor, além da própria capacidade de gestão de todas as suas etapas. Também é muito improvável que economicamente uma pequena empresa possa dominar todas as funções da cadeia produtiva. Tal situação acaba prejudicando o desenvolvimento estratégico da empresa e, conseqüentemente, sua competitividade dentro de um setor. Gimenez (2000) afirma que a literatura sobre estratégias em pequenas empresas é influenciada por uma abordagem de natureza empreendedora que reconhece a influência do comportamento individual do dirigente sobre o processo de formulação e implementação da estratégia. Essa abordagem preocupase em estudar a ligação entre as características pessoais do dirigente e o processo de administração estratégica utilizado nas pequenas empresas.

Contudo, Gimenez et al. (1999, p. 62), ao comentarem sobre a utilização de estratégias nas pequenas empresas, alertam para o fato de que "(...) assim como na literatura dedicada às grandes empresas, os estudos anal isados revelam uma abordagem fragmentada do processo de formação de estratégia em pequenas empresas. A maioria deles utiliza conceitos pouco abrangentes no estudo do conteúdo das estratégias".

Alguns pesquisadores têm se empenhado em descrever o processo de evolução das pequenas empresas. Covin e Slevin (1989: 75-87) desenvolveram relevante estudo a fim de detectar a influência de ambientes hostis e benignos no processo de gerenciamento estratégico de pequenas empresas. N esse estudo, ambientes hostis são aquel es que apresentam atividades econômicas precárias, acirrada competição, clima difícil e deficiência relativa de oportunidades possíveis de serem exploradas. Em contraste, os ambientes benignos configuram-se como seguros para as operações, apresentam el evados níveis de investimento, oportunidades de marketing e várias outras condições favoráveis ao incremento de negócios.

Em um ambiente de hostilidade, a sobrevivência e a prosperidade competitiva são grandes desafios para as organizações de menor porte. Isso decorre da limitação de recursos e da relativa falta de habilidade gerencial a que estão sujeitas. Essas hostilidades ambientais exigem ações das pequenas empresas no sentido de enfrentarem os efeitos del as decorrentes. N esse contexto, o gerenciamento é um fator capaz de gerar as estratégias que irão permitir, ou não, as alternativas para que essas organizações consigam adaptar-se às mutações ambientais.

Dessa forma, duas posturas estratégicas, emergem como forma de garantir a preservação das atividades das pequenas organizações. Essas posturas são influenciadas pela propensão do dirigente e são classificadas em dois tipos de postura estratégica: empreendedora e conservadora. A primeira caracteriza-se pela constante inovação de produtos e tecnologia, orienta-se por uma posição 
competitiva agressiva e por uma destemida inclinação a correr riscos. Em direção oposta, a postura conservadora caracteriza-se por uma mínima incl inação à inovação, uma cuidadosa postura competitiva e uma tímida disposição para assumir riscos.

Levando-se em consideração as características do dirigente da pequena empresa, tem-se que a sua posição é que irá influenciar a postura estratégica de sua organização. Pinheiro (1996, p. 87), apresenta um modelo de formulação de estratégias em pequenas empresas (Figura 1) no qual destaca as influências pessoais do dirigente como as mais importantes no processo - embora não exclusivas.

De acordo com o modelo exposto na Figura 1, observa-se que 0 ambiente externo à organização é importante no processo de tomada de decisão acerca das ações que a empresa deve assumir. A sua capacidade interna é um elemento de mesma importância na concepção das estratégias. Contudo, as capacidades internas e externas não são os únicos componentes que influenciam as decisões estratégicas nas pequenas empresas. Os dirigentes dessas organizações geralmente imprimem sua marca pessoal, agindo como um filtro para a formulação de ações e decisão estratégica. Isso ocorre porque comumente a decisão nessas organizações está central izada na figura de seu proprietário. Essa influência é importante nas primeiras etapas de vida da empresa, uma vez que a figura do dono e sua dedicação ao negócio têm uma importância crucial para a sua prosperidade.

Figura 1 - Formulação de estratégias em pequenas empresas.

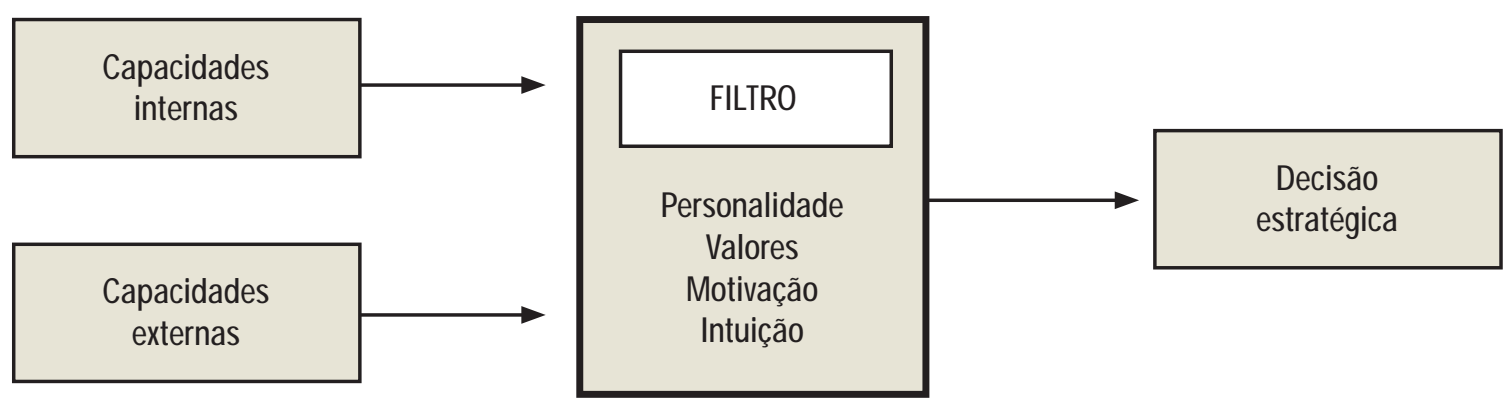

Fonte: Pinheiro (1996, p. 87). 
jetivo principal a descrição de al go e apenas relatar como ocorrem certos fenômenos ou como se comportam certas variáveis em determinada situação, sendo orientada por hipóteses teóricas preestabelecidas sobre o fenômeno estudado (M al hotra, 2001).

Como desenho mais apropriado, optou-se por uma survey interseccional, na qual os dados são coletados em um único momento de uma amostra selecionada para descrever a relação entre variáveis em uma população na mesma ocasião (Babbie, 1999). 0 instrumento utilizado para coleta de dados foi um questionário composto por 41 questões distribuídas da seguinte maneira: (1) caracterização do respondente e da empresa (T1 a T6); (2) escala referente aos aspectos da formação da estratégia (Q1 a Q 32); e (3) avaliação subjetiva do desempenho da empresa (P33 a P35).

A amostra utilizada foi não-probabilística e por julgamento dos pesquisadores. Foram procurados micro e pequenos empresários de diferentes cidades do centro-oeste do estado de Minas Gerais (Arcos, Formiga, Lagoa da Prata, Bambuí, Luz, Pains, Bom Despacho, Santo Antônio do Monte). A seguir eles foram convidados a participar da pesquisa por grupos de alunos do 60 período do curso de Administração da PUC M inas, em Arcos, que haviam sido devidamente treinados para essa abordagem e para a coleta dos dados.

Foram respondidos, na presença dos pesquisadores, os 50 questionários efetivamente utilizados na pesquisa. Os dados coletados foram tabulados e submetidos a análises uni- e multivariadas com o auxílio do SPSS (Statistical Package for the Social Science). Utilizou-se a regressão linear múltipla para se entenderem as relações existentes entre variáveis do processo de formação da estratégia consideradas no estu do e o desempenho, e ainda a análise discriminante para determinar os indicadores que diferenciam os grupos de empresas com desempenho acima e abaixo da média.

\section{Operacionalização das variáveis}

No que tange às variáveis relativas à formação da estratégia, o questionário foi redesenhado a partir da versão original construída por Meirelles (2003). Com o objetivo de simplificar a coleta, tendo em vista as características dos respondentes e o caráter ainda exploratório da pesquisa, a adaptação do instrumento consistiu na exclusão de 18 itens (ou perguntas) e na utilização de escalas do tipo Likert - não de 7, mas de 5 pontos (de Discordo totalmente a Concordo totalmente).

A decisão sobre quais seriam os itens a serem descartados foi orientada por dois critérios básicos. 0 pri- meiro, de caráter subjetivo, foi o julgamento de especialistas (Malhotra, 2001), incorporados, nesse caso, na figura de dois professores da área de Estratégia e Aprendizagem Organizacional, mestres e doutorandos em Administração, assessorados por alunos de 60 período. 0 procedimento consistiu em organizar, em sala de aula, um painel para a discussão da validade de face (de conteúdo) da escala original, tendo em vista a sua replicação em um contexto bastante diferente. A preocupação principal estava na clareza e precisão dos termos, de modo que os respondentes pudessem compreender e responder adequadamente às questões. 0 segundo critério, de caráter objetivo, derivou da análise dos resultados obtidos por Meirelles (2003), autor esse que já apontava para a possibilidade de exclusão ou de junção de itens "indiferentes", nos quais não foi constatada diferença significativa entre 0 que 0 autor chamou de estratégias satisfatórias e estratégias insatisfatórias.

Com relação à escala de avaliação subjetiva do desempenho das empresas pelos respondentes, tomaramse como referência os trabalhos de Pelham e Wilson (1996), Dess e Robinson (1984) e Perin e Sampaio (1999), os quais que têm mostrado relações positivas e significativas entre medidas objetivas e subjetivas de desempenho. Utilizaram-se nesse quesito três questões que tinham como referência de tempo um período de três anos: na primeira, o respondente foi solicitado a dar uma nota (de 0 a 10) para o desempenho geral; na segunda, ele apontava, em uma escala do tipo Likert de 5 pontos, como foi o desempenho geral da empresa em relação às suas expectativas (de Muito abaixo do esperado a M uito acima do esperado); e na terceira, também em uma escala do tipo Likert de 5 pontos, o empresário comparava o desempenho geral da sua empresa com 0 de aos seus principais concorrentes (de Muito pior do que os concorrentes a Muito melhor do que os concorrentes). 0 detal hamento dos itens das escalas utilizados para operacionalizar as dimensões tratadas na análise é apresentado no Quadro 1.

U ma medida global para o desempenho geral percebido foi cal culada a partir da média aritmética simples das respostas dadas às questões Q 33, Q 34 e Q 35, que funcionou com seu indicador. Assim, para um respondente genérico $(\mathrm{Rn})$, por exemplo, calculamos: Desempenho $=\frac{\frac{Q 33}{2}+Q 34+Q 35}{3}$. A divisão de Q33 - que vai de 0 a 10 - por dois serviu ao propósito de compatibilizá-la com as escalas de Q34 e Q35 - que variam de 1 a 5. Poderíamos ter, nesse caso, um problema com valores entre 0,00 e 1,99 na Q33, os quais, quando divididos por 2 , não teriam correspon- 


\section{ARTIGOS •FORMAÇÃO DE ESTRATÉGIA NAS MICRO E PEQUENAS EMPRESAS: UM ESTUDO NO CENTRO-OESTE MINEIRO}

Quadro 1 - Indicadores utilizados no trabalho.

\section{QUESTÕES UTILIZADAS}

Q1) A estratégia foi decidida em um processo formal de análises, discussões e, por fim, de escolha.

Q2) A estratégia foi decidida dentro do processo periódico de planejamento estratégico da empresa.

Q3) Na elaboração da estratégia, foram obtidas contribuições de vários setores da empresa que seriam afetados pela estratégia, antes de decidir pela sua adoção.

Q4) A estratégia foi exaustivamente discutida antes de ser adotada.

Q5) É difícil dizer como surgiu a idéia que afinal gerou a estratégia.

Q6) A idéia da estratégia já existia, antes de sua adoção formal, na mente de um ou mais componentes da diretoria/ gerência.

Q7) Criatividade e intuição foram essenciais na formulação da estratégia.

Q8) Na hora da decisão, o poder de grupo e indivíduos pesou mais que as informações objetivas.

Q9) Mais de uma alternativa de ação foi avaliada antes de se decidir pela adoção da estratégia.

Q10) As alternativas estratégias foram traduzidas em termos financeiros para tomar a decisão estratégia mais objetiva.

Q11) Análises criteriosas/ cuidadosas/ detalhadas do ambiente externo orientaram a decisão de adotar a estratégia.

Q12) A decisão de adotar a estratégia foi orientada por uma análise de como os vários fatores e forças do ambiente externo tenderiam a se comportar no futuro.

013) A decisão de adotar a estratégia foi orientada por uma avaliação dos prováveis movimentos que os concorrentes fariam em relação à estratégia.

Q14) A evolução do ambiente externo, após a adoção da estratégia, ocorreu dentro das expectativas.

Q15) 0 conhecimento já acumulado na empresa sobre o mercado foi mais importante para a decisão de adotar a estratégia que pesquisas e analises formal.

Q16) À época da adoção da estratégia, as opções estratégias eram limitadas, portanto pode-se dizer que a alternativa adotada praticamente se impôs.

Q17) A estratégia adotada visava o aproveitamento de oportunidade percebida no ambiente de atuação da empresa.

Q18) A estratégia adotada visava neutralizar ameaça percebida no ambiente de atuação da empresa.

019) Mesmo depois de escolhida a estratégia e iniciada sua implantação, continuou-se monitorando o ambiente externo, para que fosse possível verificar se a estratégia continuava adequada.

Q20) A avaliação das habilidades e competências internas (pontos fortes e pontos fracos) da empresa orientou a escolha da estratégia.

Q21) A estratégia, uma vez concebida, foi desdobrada em metas, planos de ação, orçamentos.

Q22) Estabeleceu-se uma agenda de avaliações para acompanhar a implantação da estratégia

Q23) A estratégia, uma vez decidida, foi devidamente comunicada a todos os que deveriam compreendê-la e segui-la.

Q24) Há uma distancia entre a estratégia formulada/ pensada inicialmente e a que foi efetivamente implantada.

Q25) As pessoas que apoiaram a decisão estratégia também mostraram firmeza na hora de implementá-la.

Q26) Cumpriu-se a agenda de acompanhamento e avaliação da implementação da estratégia.

Q27) A estratégia representou mudanças em relação às estratégias até então vigentes na empresa.

Q28) Pelo menos inicialmente, a idéia da estratégia foi recebida com desconfiança por alguns daqueles que participavam da decisão de adotá-la.

Q29) A estratégia exigia mudanças nas competências e habilidades que a empresa possui até então.

Q30) A estratégia implicava mudanças nos padrões tecnológicos até então adotados pela empresa.

Q31) A estratégia teve como referencia ações ou estratégias de competidores.

Q32) A estratégia representou uma mudança inovadora em relação ao que o mercado vinha praticando até então. 
dência direta. No entanto, a análise das respostas dadas a essa questão mostra que o valor mínimo obtido na amostra é 5 .

\section{RESULTADOS E DISCUSSÃO}

\section{Análise de regressão}

De acordo com Hair et al. (2005), a análise de regressão múltipla é uma técnica estatística utilizada para analisar a relação entre uma única variável dependente (critério) e diversas variáveis independentes (preditoras) . 0 conjunto de variáveis independentes ponderadas compõea variável estatística de regressão ( conhecida como equação ou modelo de regressão), uma combinação linear das variáveis independentes que melhor prevê a variável dependente. Os pesos (coeficientes $\beta$ ) denotam a contribuição relativa das variáveis independentes para a previsão ou explicação geral, e facilitam a interpretação sobre a influência de cada variável.

Pode-se determinar a intensidade da relação estipulada pela equação de regressão múltipla utilizando-se o coeficiente de correlação múltipla R2, que também é chamado de coeficiente de determinação múltipla e/ ou de R2 ajustado - ajustado para o número de variáveis independentes e para o tamanho da amostra. Esse coeficiente varia entre 0 e 1 e significa a proporção da variação total na variável dependente, a qual é explicada pelo modelo de regressão construído - quanto mais próximo de 1, maior o ajuste da função de regressão aos dados (Malhotra, 2001).

N este estudo foi utilizado um modelo de regressão linear múltipla, com o objetivo de investigar em que grau cada uma das 32 questões influencia o desempenho percebido das empresas. Foi realizada uma análise de variância (AN OVA) para verificar se as variáveis independentes influenciam linearmente a variável dependente. 0 resultado da ANOVA foi bastante significativo $(0,000)$, o que nos permite inferir que há evidências de que o model o pode se adequar ao estudo proposto.

Apesar de um ajuste apenas moderado $\left(\mathrm{R}_{\text {ajustado }}=\right.$ $0,524)$, como se constata na Tabela 1 , o modelo de regressão e os coeficientes são significativos, o que, tendo em vista as peculiaridades da pesquisa em ciências sociais, nos permite persistir na análise para extrair conclusões dotadas de relevância prática. A equação revela a magnitude e as direções (positivas e negativas) das relações entre as variáveis Q21, Q 32 e Q28 - as quais se mostraram significativas dentre as 32 utilizadas - e o desempenho geral percebido das empresas. Esses indicadores são capazes de explicar $52,4 \%$ da variância na variável desempenho. Estabelecidas as relações, partiu-se para uma segunda etapa de análise dos dados, cujo objetivo fundamental foi identificar quais seriam as variáveis - ou indicadores operacionais - mais fortemente relacionadas com graus elevados de desempenho. Para isso, Iançou-se mão da análise discriminante.

\section{Análise discriminante}

Segundo Hair et al. (2005), a análise discriminante éuma técnica estatística multivariada utilizada quando se quer estabelecer relações entre uma variável dependente nãométrica e variáveis independentes métricas. Por meio da análise discriminante é possível identificar quais variáveis são mais relevantes para explicar as diferenças entre os grupos que sejam heterogêneos num contexto, mas homogêneos entre si. Trata-se da obtenção de uma função, que é a combinação linear de duas ou mais variáveis independentes, que discrimina melhor entre os grupos definidos a priori.

Dois métodos computacionais são normalmente utilizados na determinação da função discriminante: a estimação simultânea - quando se quer incluir todas as variáveis independentes na anál ise; e a estimação passo a passo (stepwise), utilizada no presente trabal ho - quando a intenção é identificar as variáveis com maior poder de discriminação. Nesse caso, as variáveis independentes são analisadas uma a uma, iniciando com a que melhor

Tabela 1 - Modelo de regressão $\quad$ [Desempenho] $=\beta$ o $+\beta 1 .[Q 1]+\beta 2 .[Q 2]+(\ldots)+\beta 32$.[Q32] + e.

\begin{tabular}{|c|c|c|c|c|c|}
\hline $\begin{array}{c}\text { VARIÁVEL } \\
\text { DEPENDENTE }\end{array}$ & $\begin{array}{c}\text { VARIÁVEL } \\
\text { INDEPENDENTE }\end{array}$ & $\beta$ PADRONIZADO & $\begin{array}{c}\text { SIG. } \\
\text { (COEFICIENTES) }\end{array}$ & $\begin{array}{c}\text { R }^{2} \\
\text { AJUSTADO }\end{array}$ & $\begin{array}{c}\text { SIG. } \\
\text { (MOD. REGRESSÃO) }\end{array}$ \\
\hline \multirow{2}{*}{ Desempenho } & Q21 & 0,494 & 0,000 & & \\
& Q32 & 0,335 & 0,004 & 0,524 & 0,000 \\
\hline
\end{tabular}


discrimina dentre todas as outras (Malhotra, 2001). 0 primeiro passo para a realização de uma análise discriminante é a definição dos grupos que se pretende estudar, isto é, a definiçaão da variavel categórica (não-métrica) a ser utilizada. 0 parâmetro utilizado aqui para a classificação dos grupos foi o nível de desempenho geral percebido. Depois de calculada a média global da variável [desempenho], considerando o conjunto dos 50 respondentes da amostra, cada uma das empresas foi classificada da seguinte forma:

Desempenho abaixo da média - Desempenho global percebido da empresa menor ou igual à média global, considerando as 50 empresas participantes da pesquisa

Desempenho acima da média - Desempenho global percebido da empresa mai or do que média gl obal, considerando as 50 empresas participantes da pesquisa
A Tabela 2 aponta que há diferença estatisticamente significativa entre os grupos de empresas com desempenho acima e com desempenho abaixo da média.

Essa constatação nos dá subsídios para seguir adiante em busca de quais, dentre todas as 32 variáveis descritas no Quadro 1 e consideradas como variáveis independentes métricas, têm o poder de discriminar entre desempenho acima e abaixo da média. Os resultados da análise discriminante mostram que 3 variáveis independentes (Q32, Q21 e Q28) discriminam a variável dependente (desempenho - acima ou abaixo da média). É interessante notar que essas são as mesmas variáveis que compuseram o modelo de regressão linear múltipla, emergindo como principais preditoras do desempenho. A Tabela 3 apresenta a estatística W ilk's Lambda e o nível de significância de cada variável selecionada.

A análise discriminante processou uma única função discriminante formada a partir das variáveis estu-

Tabela 2 - One WayANOVA para diferentes níveis de desempenho.

\begin{tabular}{|c|c|c|c|c|c|}
\hline & $\mathrm{N}$ & \multicolumn{2}{|c|}{ MÉDIA } & \multicolumn{2}{|c|}{ DESVIO PADRÃO } \\
\hline Desempenho abaixo da média & 22 & \multicolumn{2}{|c|}{$-0,921$} & \multicolumn{2}{|c|}{0,694} \\
\hline Desempenho acima da média & 28 & \multicolumn{2}{|c|}{0,724} & \multicolumn{2}{|c|}{0,453} \\
\hline Total & 50 & \multicolumn{2}{|c|}{0,000} & \multicolumn{2}{|c|}{1,000} \\
\hline & $\begin{array}{l}\text { SOMA DOS } \\
\text { QUADRADOS }\end{array}$ & $\begin{array}{l}\text { GRAUS DE } \\
\text { LIBERDADE }\end{array}$ & $\begin{array}{l}\text { QUADRADOS } \\
\text { MÉDIOS }\end{array}$ & $\mathrm{F}$ & SIG. \\
\hline Entre grupos & 33,333 & 1 & 33,333 & 102,122 & 0,000 \\
\hline No grupo & 15,667 & 48 & 0,326 & & \\
\hline Total & 49,000 & 49 & & & \\
\hline
\end{tabular}

Tabela 3 - Resultados da análise discriminante stepwise

\begin{tabular}{|c|c|c|c|}
\hline STEP & ENTRADA DA VARIÁVEL & WILK'S LAMBDA & SIG. \\
\hline 1 & $\begin{array}{l}\text { Q32 } \\
\text { A estratégia representou uma mudança inovadora em relação ao que o mercado vinha } \\
\text { praticando até então }\end{array}$ & 0,672 & 0,000 \\
\hline 2 & $\begin{array}{l}\text { Q21 A estratégia, uma vez concebida, foi desdobrada em metas, planos de ação, } \\
\text { orçamentos }\end{array}$ & 0,681 & 0,000 \\
\hline 3 & $\begin{array}{l}\text { Q28 Pelo menos inicialmente, a idéia da estratégia foi recebida com desconfiança por al- } \\
\text { guns daqueles que participavam da decisão de adotá-la }\end{array}$ & 0,636 & 0,000 \\
\hline
\end{tabular}


dadas cujas principais características estão descritas na Tabela 4.

Os coeficientes de correlação canônica encontrados, inclusive as três variáveis selecionadas pelo procedimento stepwise, indicam que as funções discriminantes possuem al to grau de significância, mas que o poder de ajuste aos dados é apenas mediano. No entanto, considerando tratar-se de uma pesquisa em ciências sociais, e que certamente existe uma série de outras variáveis não previstas no modelo que poderiam interferir nos resultados, pode-se afirmar que os dados coletados e as funções discriminantes obtidas são importantes elementos para o entendimento da questão de pesquisa levantada e contribuirão para futuros estudos.

A Tabela 5 exibe os coeficientes das funções discriminantes canônicas padronizadas. Essa tabela mostra que as variáveis Q21 - a estratégia, uma vez concebida, "foi desdobrada em metas, planos de ação, orçamentos" - e Q32 "a estratégia representou uma mudança inovadora em relação ao que o mercado vinha praticando até então" - , são as que têm uma contribuição maior na função discriminante $1(0,656$ e 0,610 , respectivamente). $N$ a seqüência, emerge a variável Q28 - "pelo menos inicialmente, a idéia da estratégia foi recebida com desconfiança por alguns daqueles que participavam da decisão de adotá-la", com uma contribuição negativa, indicando uma discri- minação em sentido contrário (invertido - maior Q28, desempenho abaixo da média; menor Q28, desempenho acima da média) ao das demais variáveis. Prognosticadores com coeficientes padronizados relativamente grandes contribuem mais para o poder discriminatório da função (Malhotra, 2001).

A partir das funções discriminantes, foram calculados os escores discriminantes para cada observação feita,que permitira, assim, classificar se um valor observado nas variáveis independentes é proveniente de uma empresa com desempenho acima ou abaixo da média. Para tanto, utiliza-se a fórmula geral da função discriminante, cujos coeficientes estão descritos na Tabela 6.

A Tabela 7 apresenta os resultados de classificação obtidos a partir da função discriminante. Pode-se verificar que o percentual de elementos do grupo com desempenho abaixo da média, corretamente classificados após a aplicação da análise discriminante, é de $72,7 \%$, enquanto o percentual de acerto para o grupo com desempenho acima da média é de $85,7 \%$. Portanto, em média, 80,0\% dos elementos foram corretamente classificados após a aplicação da análise discriminante.

Segundo Hair et al. (2005), para averiguar se esse percentual de classificação é satisfatório pode-se aplicar um teste estatístico adicional que verifique o poder discriminatório da matriz de classificação quando comparada

Tabela 4 - Teste Wilk's Lambda.

\begin{tabular}{|c|c|c|c|c|c|c|c|}
\hline $\begin{array}{c}\text { TESTE DA } \\
\text { FUNÇÃO }\end{array}$ & $\begin{array}{c}\text { AUTO } \\
\text { VALOR }\end{array}$ & $\begin{array}{c}\text { \% VARIÂNCIA } \\
\text { EXPLICADA }\end{array}$ & WILK'S LAMBDA & CHI-SQUARE & DF & SIG. & $\begin{array}{c}\text { COEF. CORREL. } \\
\text { CANÔNICA }\end{array}$ \\
\hline 1 & 0,745 & 100 & 0,573 & 25,898 & 3 & 0,0001 & 0,653 \\
\hline
\end{tabular}

Tabela 5 - Coeficientes da função discriminante canônica padronizada.

\section{VARIÁVEIS INDEPENDENTES}

Q21 A estratégia, uma vez concebida, foi desdobrada em metas, planos de ação, orçamentos

Q28 Pelo menos inicialmente, a idéia da estratégia foi recebida com desconfiança por alguns daqueles que participavam da decisão de adotá-la

Q32 A estratégia representou uma mudança inovadora em relação ao que o mercado vinha praticando até então.
COEFICIENTES PADRONIZADOS DA FUNÇÃO

1

0,656

$-0,503$

0,610 
a um modelo al eatório. Trata-se da estatística Press's Q, dada pela fórmula a seguir:

$$
\text { Press's } Q=\left[N-\left(n_{*} K\right)\right]^{2} / N_{*}(K-1)
$$

onde: $\mathrm{N}=$ número total da amostra; $\mathrm{n}=$ número de observações corretamente classificadas e $\mathrm{K}=$ número de grupos.

Substituindo os valores na fórmula ( $N=50, n=40, K=$ 2 ), chega-se a um valor para a estatística $Q$ igual a 18,00 . Como esse valor excede, e muito, o valor crítico $(6,63$ a um nível de significância de 1\%), pode-se concluir que a discriminação dos clientes nos três grupos foi significativamente mel hor do que uma distribuição al eatória. Muito embora o percentual de elementos corretamente classificados, após a aplicação da técnica $(80,0 \%)$, possa parecer baixo, na aplicação em casos práticos da técnica de anál ise discriminante em ciências sociais é comum encontrar valores dessa ordem (Andreassi e Sbragia, 2001).

\section{CONSIDERAÇÕES FINAIS}

Estudar o processo estratégico das micro e pequenas empresas (M PEs) do centro-oeste de M inas Gerais mostrouse um desafio interessante. Se, por um lado, a compreen- são da estratégia de tais organizações pode ser inferida a partir dos estudos em estratégias desenvolvidos na atualidade, já que em larga medida as general izações são desenvolvidas a partir das principais correntes da área, por outro, pensa-se que é necessária uma problematização mais direcionada, como a proposta deste estudo: afinal, o desenvolvimento de conhecimento sobre a área, predominantemente voltado para organizações de médio e grande porte, é passível de generalização para as micro e pequenas empresas brasileiras?

Acredita-se que a realidade das organizações do centro-oeste de Minas Gerais pode expressar al guns aspectos específicos que carecem de uma atenção maior. Se, por um lado, a noção de administração estratégica pode soar general izável, por outro, corre o risco de deixar de captar realidades específicas, como a das M PEs localizadas pelo Brasil "afora". N esse sentido, a investigação realizada por este estudo pretendeu diminuir a distância entre os conceitos formulados acerca da administração estratégica e a realidade de organizações brasileiras - em particular, aquelas localizadas no interior do país. A utilização de variáveis quantitativas que ajudam a expressar aspectos específicos dessa realidade contribui para o avanço na compreensão do processo de formação de estratégias de tais MPEs.

Três variáveis aparecem com destaque e de maneira re-

Tabela 6 - Função discriminante linear de Fisher.

\section{VARIÁVEIS}

DESEMPENHO

\begin{tabular}{|c|c|}
\hline $\begin{array}{c}\text { ABAIXO DA } \\
\text { MÉDIA }\end{array}$ & $\begin{array}{c}\text { ACIMA DA } \\
\text { MÉDIA }\end{array}$ \\
\hline$-0,727$ & 0,529 \\
\hline 0,442 & $-0,418$ \\
\hline$-0,667$ & 0,518 \\
\hline$-1,284$ & $-0,857$ \\
\hline
\end{tabular}

Tabela 7 - Matriz de classificação.

\begin{tabular}{|c|c|c|c|}
\hline \multirow{2}{*}{ GRUPO ORIGINAL } & NÚMERO DE CASOS & \multicolumn{2}{|c|}{ NÍVEL DE DESEMPENHO } \\
\cline { 2 - 4 } & 22 & ABAIXO DA MÉDIA & ACIMA DA MÉDIA \\
\hline Abaixo da média & 28 & $\mathbf{1 6}(\mathbf{7 2 , 7} \%)$ & $6(27,3 \%)$ \\
\hline Acima da média & $4(14,3 \%)$ & $\mathbf{2 4}(\mathbf{8 5}, \mathbf{7} \%)$ \\
\hline
\end{tabular}


corrente quando investigamos os preditores do desempenho e os indicadores que diferenciam desempenhos acima e abaixo da média. Isso se deve, entre outras coisas, ao fato de as técnicas estatísticas utilizadas, até certo ponto, se sobreporem. 0 interessante, neste caso, é a aparente contradição entre as variáveis Q21 ("a estratégia, uma vez concebida, foi desdobrada em metas, planos de ação, orçamentos") e Q32 ( "a estratégia representou uma mu dança inovadora em relação ao que o mercado vinha praticando até então"). Temos, então, uma variável privilegiada em processos de formação de estratégia mais racionais, deliberados, objetivos e formais, e somos também obrigados a reconhecer, ao mesmo tempo, nesse mesmo processo, um quê de inovação e mudança. 0 desempenho acima da média, ao que parece, pelo menos para esse tipo de empresa, é resultado de uma receita balanceada desses dois ingredientes.

Esse tipo de constatação, entretanto, não é novidade. Levá-la a sério e considerá-la seriamente na teorização acerca da estratégia nas organizações é que parece ser o problema. Vasconcelos (2001), por exemplo, já havia chamado a atenção para a "a síndrome do ornitorrinco". Em vez dos animais previstos por M intzberg et al. (2000) em seu Safári de Estratégia, é provável que encontremos criaturas realmente estranhas quando compararmos os model os teóricos com a prática da estratégia. Se, por um lado, a mente dos estrategistas parece funcionar bem com inconsistências, paradoxos, paradigmas múltiplos e modelos conflitantes; por outro, as teorias são freqüentemente simplificadoras demais para dar conta de todas as complexidades, econômicas, técnicas e humanas, da estratégia empresarial (Vasconcelos, 2001, p.13).

Portanto, o escopo de análise da administração estratégica deve ser ampliado para dar conta de um processo que envolva a construção simultânea de estabilidade e mudança estratégicas (Hendry e Seidl, 2003; Wilson e Jarzabkowski, 2004; Jarzabkowski, 2004), procurando lidar com a complexa relação entre aquilo que é intencional e o que é emergente na construção das estratégias (Hendry, 2000; Jarzabkowski, 2005). 0 próprio M intzberg (1987) já havia reconhecido a necessidade de se balancear del iberação e emergência. Uma estratégia puramente deliberada reduziria as possibilidades de aprendizagem e mudança, enquanto uma estratégia puramente emergente poderia levar à fal ta de controle do processo estratégico. É razoável imaginar que "boa" parte dos resultados obtidos pela organização seja fruto de ações emergentes. No entanto, não se pode ignorar que os estrategistas precisam se envolver também em uma série de atividades formais e sistemáticas.
Nesse sentido mais do que "simplesmente" navegar recorrendo sempre aos mapas e às representações "racionais" que fazemos do mar/território, para nos dizer onde estamos e para onde vamos, é preciso "sentir" o caminho, ajustando a nossa rota ao fluxo contínuo e mutável das ondas, do vento e das correntes que também estão sempreem movimento. 0 estrategista, nesse sentido, talvez se identifique menos com a figura do técnico, por exemplo, e mais com a figura do jogador de futebol. Apesar do esquema ( pré-) definido, é dentro do campo, no "cal or do jogo", que as coisas acontecem. 0 jogador, totalmente imerso no jogo, e sob a luz dos holofotes, precisa continuamente se ajustar e tentar responder às ações "dos outros" - sejam seus companheiros, adversários, técnico, juiz, auxiliares, torcida, condições do campo e do tempo -, tendo, para isso, na maior parte das vezes, não mais do que um "piscar de olhos" (Chia e Holt, 2006).

\section{REFERÊNCIAS}

ALVES, R. C. Construção de estratégias na indústria avícola: um estudo de caso numa empresa produtora de carne de frango. Dissertação (M estrado em Administração). Belo Horizonte. FEAD Minas, 2006.

ANDREASSI, T.; SBRAGIA, R. Fatores determinantes do grau de inovatividade das empresas: um estudo utilizando a técnica de análise discriminante. In: ENCONTRO NACIONAL DA ASSOCIAÇÃO DE PÓSGRADUAÇÃO EM ADMINISTRAÇÃO, 23., Campinas, 2001. Anais. Campinas: ANPAD, 2001.

ANSOFF, H. I.; MCDON NELL, E. J. Implantando a administração estratégica. 2. Ed. São Paulo: Atlas, 1993.

BABBIE, E. M étodos de pesquisas de survey. Belo Horizonte: Editora UFMG, 1999.

BALESTRIN, A; VARGAS, L. M. Redes horizontais de cooperação como estrutura favorável para o desenvolvimento das PMEs. In: ENCONTRO NACIONAL DA ASSOCIAÇÃO DE PÓS-GRADUAÇÃO EM ADMINISTRAÇÃO, 27., 2003, Atibaia. Anais. Atibaia: AN PAD, 2003.

CASSAROTO FILHO, N.; PIRES, L. H. Redes de pequenas e médias empresas e o desenvolvimento local. São Paulo: Atlas, 1998.

CHANDLER, A. What is a firm? A historical perspective. European Economic Review, v. 36, p. 483-494, 1992.

CHER, R. A gerência das pequenas e médias empresas. São Paulo: Maltese, 1990.

CHIA, R.; HOLT, R. Strategy as practical coping: a Heideggerian perspective. Organization Studies, v. 27, n. 5, p. 635-655, 2006. 


\section{ARTIGOS •FORMAÇÃO DE ESTRATÉGIA NAS MICRO E PEQUENAS EMPRESAS: UM ESTUDO NO CENTRO-OESTE MINEIRO}

COVIN, J. G.; SLEVIN, D. P. Strategic management of small firms in hostile and benign environments. Strategic Management Journal, v. 10, n. 1, p. 75-87, 1989.

DESS, G. G.; ROBINSON, R. B. JR. M easuring organizational performance in the absence of objective measures: the case of the privately-held firm and conglomerate business unit. Strategic M anagement Journal, v. 5, n. 3, p. $265-273,1984$.

FIGUEIREDO, N. G; MARTINELLI, D. P. Inovação e exportação em pequenas e médias empresas (PME): uma pesquisa em andamento. In: ENCONTRO NACIONAL DA ASSOCIAÇÃO DE PÓS-GRADUAÇÃO EM ADMINISTRAÇÃO, 26., Salvador, 2002. Anais. Salvador: ANPAD, 2002.

GIMENEZ, F. A. P.; PELISSO N, C.; HAYASHI JR., P.; KRÜGER, E. Estratégia em pequenas empresas: uma aplicação do modelo de Miles e Snow. Revista de Administração Contemporânea, v. 3, n. 2, p. 53-74, mai./ago. 1999.

GIMENEZ, F. A. P. 0 estrategista na pequena empresa. Maringá. Edição do autor, 2000, v.1, 176 p.

GOOLD, M.; CAM PBELL, A. As melhores maneiras de formular estratégias. In: MONTGOMERY, C. A. Estratégia: a busca da vantagem competitiva. 2. Ed. Rio de Janeiro: Campus, p. 349-362, 1998

HAIR JR., J. F.; ANDERSO N, R. E.; TATHAN, R. L.; BLACK, W. C. Análise multivariada de dados. Porto Alegre: Bookman, 2005.

HENDERSON, B. D. As origens da estratégia. In: MONTGOMERY, C. A. Estratégia: a busca da vantagem competitiva. 2. Ed. Rio de Janeiro: Campus, p. 3-11, 1998.

HENDRY, J. Strategic decision-making, discourse, and strategy as social practice. Journal of M anagement Studies, v. 37, n. 7, p. 955-977, 2000.

HENDRY, J.; SEIDL, d. The structure and significance of strategic episodes: social systems theory and the routine practices of strategic change. Journal of M anagement Studies, v. 40, n. 1, p. 175-196, 2003.

JARZABKOWSKI, P. Strategy as Practice: An Activity-based Approach. London: Sage Publications, 2005.

JARZABKOWSKI, P. Strategy as practice: recursiveness, adaptation, and practices-in-use. Organization Studies, v. 25, n. 4, p. 529-560, 2004.

LAKATOS, E. M. Sociologia da Administração. São Paulo: Atlas, Cap. 8, p. 198-215, 1997.

MALH OTRA, N. K. Pesquisa de marketing: uma orientação aplicada. 3. Ed. Porto Alegre: Bookman, 2001.

MEIRELLES, A. de M. et al. Uma abordagem para estratégia utilizando analogias. In: ENCONTRO NACIONAL DA ASSOCIAÇÃO DE PÓSGRADUAÇÃO EM ADMINISTRAÇÃO, 24., Florianópolis, 2000. Anais. Florianópolis: AN PAD, 2000.

MEIRELLES, A. de M. A formação de estratégia no sistema bancário brasileiro: modelo teórico e evidências empíricas. Tese (Doutorado em Administração). Belo Horizonte. UFM G/CEPEAD, 2003.
MINTZBERG, H. A organização empreendedora. In: MINTZBERG, H.; QUINN, J. B. 0 processo da estratégia. Porto Alegre: Bookman, p. 231$239,2001$.

MINTZBERG, H.; AHLSTRAND, B.; LAM PEL, J. Safári de estratégia. Porto Alegre: Bookman, 2000.

MINTZBERG, H. A criação artesanal da estratégia. In: MONTGOMERY C. A. Estratégia: a busca da vantagem competitiva. 2. Ed. Rio de Janeiro: Campus, p. 419-440, 1998.

MINTZBERG, H. Crafting strategy. Harvard Business Review, jul./ago., p.23-32, 1987.

MINTZBERG, H; AHLMSTRAND, B; LAMPEL, J. Safári de estratégia: um roteiro pela selva do planejamento estratégico. Porto Alegre: Bookman, 2000.

MINTZBERG, H; WATERS, J. Of strategies, deliberate and emergent. Strategic Management Journal, v. 6, n. 3, p. 273-293, 1985.

OHMAE, K. De volta à estratégia. In: MONTGOMERY, C. A. Estratégia: a busca da vantagem competitiva. 2. Ed. Rio de Janeiro: Campus, p. 6783, 1998.

PELHAM, A. M.; WILSON, D. T. A longitudinal study of the impact of market structure, firm structure, strategy, and market orientation culture on dimensions of small-firm performance. Journal of the Academy of Marketing Science, v. 24, n. 1, p.37-45, 1996.

PERIN , M. G.; SAM PAIO , C. H. Performance empresarial: uma comparação entre indicadores subjetivos e objetivos. In: EN CONTRO NACIONAL DA ASSOCIAÇÃO DE PÓS-GRADUAÇÃO EM ADMINISTRAÇÃO, 23., FOZ do Iguaçu, 1999. Anais. Foz do Iguaçu: AN PAD, 1999.

PINHEIRO, M. Gestão e desempenho das empresas de pequeno porte: uma abordagem conceitual e empírica. Tese (Doutorado em Administração). Faculdade de Economia, Administração e Contabilidade. São Paulo: Universidade de São Paulo, 1996.

PORTER, M. E. Competição: estratégias competitivas essenciais. Rio de Janeiro: Campus, 1999.

PORTER, M. E. Vantagem competitiva: criando e sustentando um desempenho superior. Rio de Janeiro: Campus, 1989.

PRAHALAD, C. K. A competência essencial da corporação. In MONTGOMERY, C. A. Estratégia: a busca da vantagem competitiva. Rio de Janeiro: Campus, p. 293-317, 1998.

SARAIVA, E. V. A construção de estratégias: um estudo de caso no setor de telefonia móvel. Dissertação (M estrado em Administração). Belo Horizonte. UFM G/CEPEAD, 2004.

SEBRAE. Boletim estatístico das micro e pequenas empresas. Disponível em: বhttp://www.sebrae.org.br>. Acesso em: 10 fev. 2007. 
SILVA, A. B.; PEREIRA, A. A. Fatores de influência na gestão das empresas de pequeno e médio porte da Grande Florianópolis/SC. In: ENCONTRO NACIONAL DA ASSO CIAÇÃO DE PÓS-GRADUAÇÃO EM ADMINISTRAÇÃO, 28., Curitiba, 2004. Anais. Curitiba: AN PAD, 2004.

VASCONCELOS, F. Safári de estratégia, questões bizantinas e a síndrome do ornitorrinco: uma análise empírica dos impactos da diversidade teórica em estratégia empresarial sobre a prática dos processos de tomada de decisão estratégica. In: ENCONTRO NACIONAL DA ASSOCIAÇÃO DE PÓS-GRADUAÇÃO EM ADMINISTRAÇÃO, 25., Campinas, 2001. Anais. São Paulo: Campinas, AN PAD, 2001.

WACK, P. Cenários: águas desconhecidas à frente. In: MONTGOMERY, C. A. Estratégia: a busca da vantagem competitiva. 2. Ed. Rio de Janeiro: Campus, p. 363-394, 1998.
W HITTINGTON, R. Estratégia após o modernismo: recuperando a prática. RAE-revista de administração de empresas. v. 44, n. 4, out./dez., 2004.

WHITTINGTON, R. National innovation and transnational variation. Keynote presentation, European Academy of Management. Bocconi University, Milan (Italy), abr. 2003.

WHITTINGTON, R. 0 que é estratégia? São Paulo: Thomson, 2002.

WILSON, D. C.; JARZABKOWSKI, P. Thinking and acting strategically: new challenges for interrogating strategy. European Manage M anagement Review, v. 1, n. 1, p. 14-20, 2004.

WILSON, D. C.; JARZABKOW SKI, P. Pensando e agindo estrategicamente: novos desafios para a análise estratégica. RAE-revista de administração de empresas, v. 44, n. 4, out./dez. 2004.

\section{Artigo recebido em 04.05.2007. A provado em 20.08.2007.}

\section{Leonardo Lemos da Silveira Santos}

Professor da PUC-Minas. Doutorando em Administração de Empresas pelo PPG/UFLA.

Interesses de pesquisa nas áreas de estratégia nas organizações e metodologias qual itativas de pesquisa.

E-mail: leonardo.lemos@uol.com.br

Endereço: Av. Sete de Setembro, 225, apto. 102, Divinópolis - MG, 35500-011.

\section{Ricardo César Alves}

Professor Titular da PUC-M inas. M estrado Profissional em Administração pela FEAD-M inas. Interesses de pesquisa nas áreas de estratégia nas organizações e gestão de micro e pequenas empresas.

E-mail: rialves@uai.com.br / rialves@pucminas.br

Endereço: Rua Juvenal dos Santos, 361 / 301, Luxemburgo, Belo Horizonte - MG, 30380-530.

\section{Kenneth Nunes Tavares de Almeida}

Professor das Faculdades Jorge Amado. Doutorando em Administração pela UFLA. Interesses de pesquisa nas áreas de comportamento organizacional e estratégias organizacionais.

E-mail: kennethnunes@yahoo.com.br

Endereço: Rua Catarina Fogaça, 189 / 108, Jardim Armação, Salvador - BA, 41750-120. 